\title{
Incidence and significance of coronary artery calcification
}

\author{
J. H. McCarthy and F. J. Palmer
}

From the Departments of Pathology and Radiology, Prince Henry Hospital, Sydney, and the University of New South Wales, Australia

The incidence of coronary artery calcification was studied in 65 hearts obtained at necropsy.

In cases over the age of 40 there was an incidence of coronary calcification of 79 per cent. The incidence increases with age and is slightly more common in men. These lesions occur more commonly in the left coronary circulation than the right. There is a strong association with aortic valve calcification.

Postmortem angiographic studies and microcopical examinations indicate that there is a close association between calcification and significant arterial atheroma.

It is suggested that routine fluoroscopy should include a search for coronary calcification.

Calcification of arterial atheroma occurs in the coronary arteries as it does in the remainder of the arterial tree. Such calcified coronary arteries are occasionally noted on routine chest radiographs but are difficult to distinguish from normal mediastinal structures and are easily confused with calcifications in the chest wall, lungs, or other intracardiac structures.

These lesions are more easily shown by image intensification fluoroscopy when the shape, size, position, and density of an abnormality can be viewed in high resolution in several planes and its movement relative to other structures clearly visualized. Tampas and Soule (1966) made a careful study of the cardiac outline for coronary artery calcification during 1097 routine chest screenings. The incidence of coronary calcification in patients over 40 years of age was I 5 per cent.

If coronary artery calcification were invariably associated with significant arterial stenosis or occlusion then routine chest screening with image intensification would be a simple, safe, and valuable means of detecting such patients. However, the pathological and prognostic significance of coronary calcification appears to be in some doubt. Steiner, Sutton, and Grainger (1969), writing in a widely-used radiology textbook states, 'In general there is no correlation between the degree of coronary artery calcification and the severity of coronary artery disease.' Other authors have expressed similar opinions (Adams, Abrams, and Ruttley, 1972; Vlodaver, Neufeld, and Edwards, 1972).

During 1972 we undertook a joint radiologicalpathological study into atherosclerotic coronary heart disease which was subsequently incorporated into a thesis (McCarthy, 1973). This study provided an opportunity to observe the incidence of radiologically visible coronary artery calcification and to assess its significance by further radiological and morbid anatomical studies. Sixty-five hearts removed at necropsy over an 8-month period were studied. Case selection was random and largely consecutive, and there were various causes of death, including trauma, respiratory disease, heart disease, intracranial catastrophe, and chronic renal disease. All age groups were represented but there was naturally a preponderance of older patients in an unselected necropsy series.

\section{Method}

The heart was removed intact including the proximal aorta and main pulmonary artery. Radiographs were taken in the anteroposterior and lateral planes. For hearts of normal size a focal film distance $102 \mathrm{~cm}$ was used and factors 36-40 kilovolts and 230 milliamps employed. Appropriate variations in factors were made for enlarged hearts. Non-screen film was used without a grid.

After the plain radiographs the coronary arteries were injected with radio-opaque material using a modification of Schlesinger's original method (Schlesinger, 1938). The injection mass of Laurie and Woods (1958) 


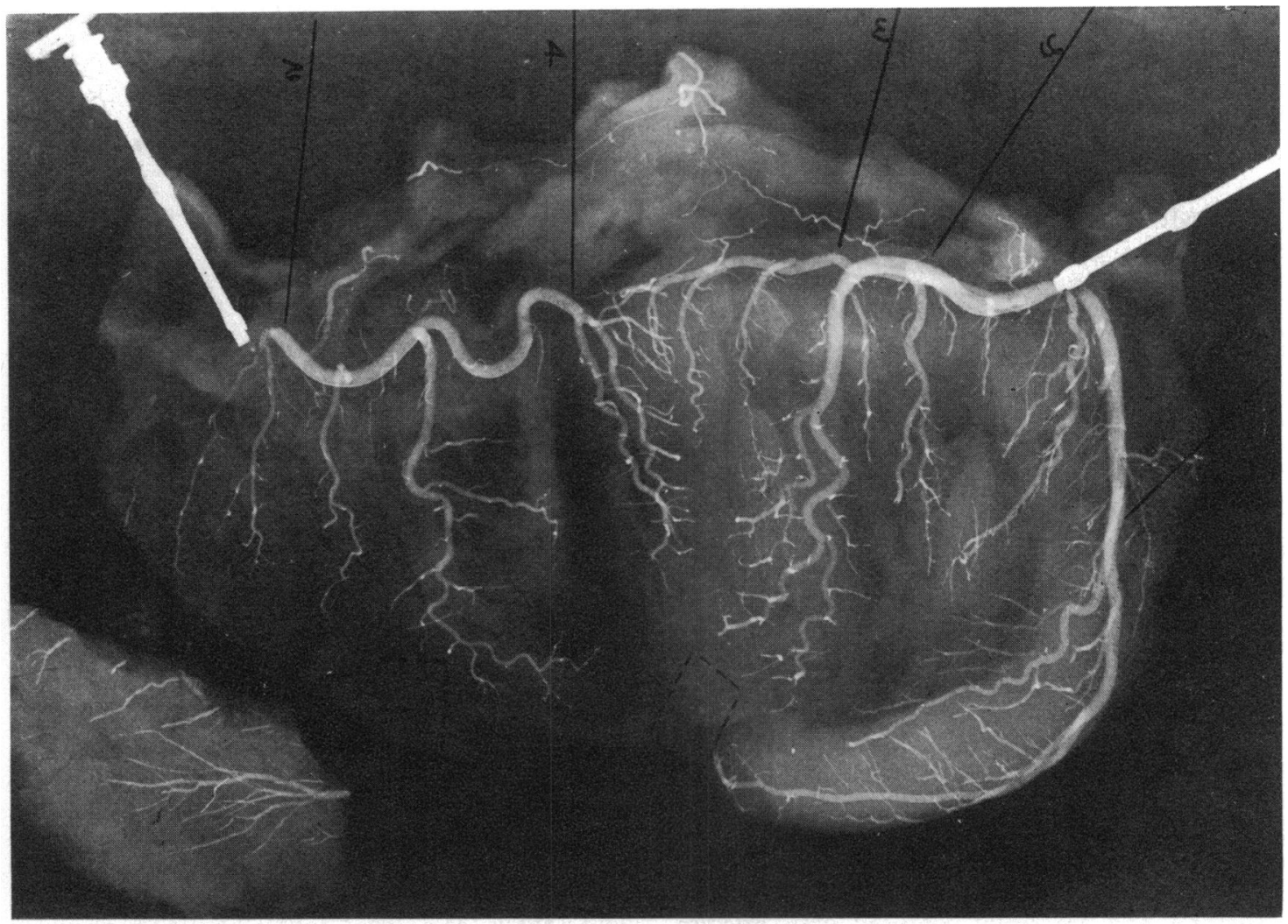

FIG. I Normal coronary arteries outlined by contrast medium. Heart 'unrolled' for optimum display.

was found to be the most satisfactory medium. Solidification of the medium was obtained by placing the heart in iced saline. Further anteroposterior and lateral radiographs were obtained before dissection.

The heart was opened by Schlesinger's method which has the virtue of 'unrolling' the heart so that the whole coronary arterial system is displayed and confusing overlap of vessels avoided (Schlesinger, 1938). Further radiographs were obtained (Fig. I).

Microscopical and histological examinations of the coronary arteries were made with particular reference to areas of apparent abnormality as indicated by the angiographic studies.

\section{Results \\ Incidence of calcification}

Coronary artery calcification was present in $4 \mathrm{I}$ $(63 \%$ ) (Fig. 2) but there were no cases with calcification under the age of 40. The incidence over the age of 40 was 79 per cent and there was seen to be a significant increase in incidence with advancing age (Table). Only 2 of the cases over the age of 60
TABLE Incidence of calcification related to age

$\begin{aligned} & \text { Age groups } \\ & \text { Whole } 40+\end{aligned}$
series

had no calcification; they were both women aged $6 \mathrm{I}$ and 70.

The incidence in men $(84 \%)$ was higher than in women $(75 \%)$. Since the average age of the men was $6 \mathrm{I}$ and of the women 58.5 , the figures are comparable and confirm the slightly higher incidence in men found in other series (Tampas and Soule, I966; Warburton et al., I968).

The calcified lesions vary in appearance. Blankenhorn and Stern (1959) defined the following types.

I) Punctate lesions: discrete opacities of varying size; may be linear, rounded, or irregular in shape. These occurred in 38 per cent. 
2) Block calcification: oblong or linear densities sufficiently large to outline the vessel, and indicating its size and direction. These occurred in 36 per cent.

3) Double calcification. Linear and punctate densities outline both sides of the vessel but not filling it completely. This type of lesion occurred in 22 per cent.

4) Incomplete double calcification. The ve:sel is outlined in one or both sides by small separate lesions of approximately $\mathrm{I} \mathrm{mm}$ diameter (Fig. 2 to 5). This occurred in only 4 per cent.

It proved difficult to classify the findings in this way as several or all types commonly occurred in the same specimen.

\section{Distribution of calcification}

Calcified lesions ranged from a single, small plaque in one major branch to extensive calcification for all major branches (Fig. 2). The left coronary system showed a tendency for earlier and more extensive calcification than the right coronary artery and there were no examples of extensive calcification restricted to the right coronary artery. Most cases with pronounced calcification showed involvement of all major vessels (Fig. 2). The incidence of predominant

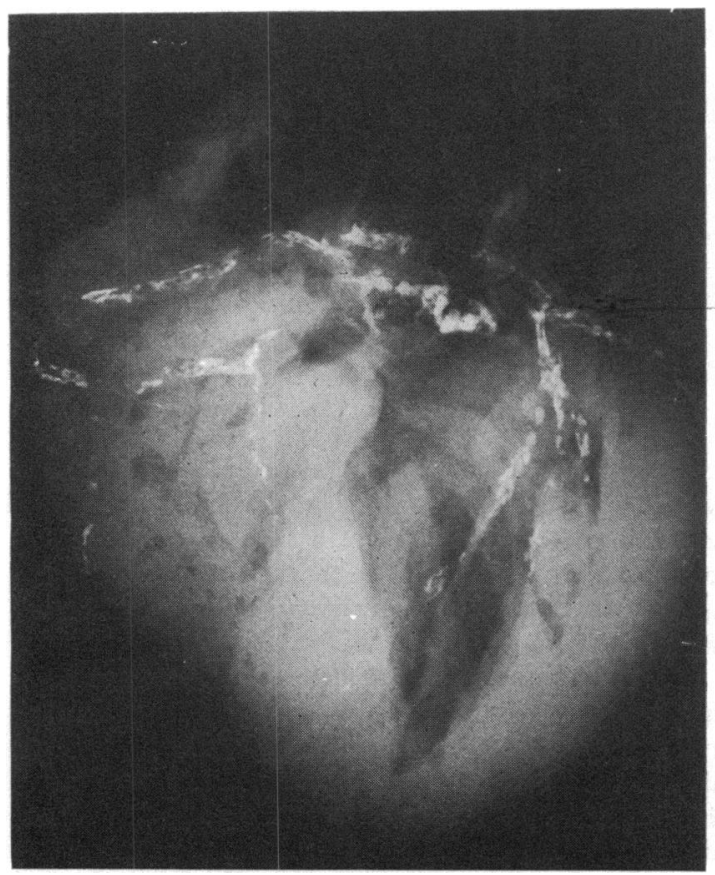

FIG. 2 Gross coronary calcification involving all proximal vessels. vessel involvement was as follows: left coronary and/or anterior descending, 47 per cent; right coronary, 30 per cent; circumflex, 23 per cent.

The proximal $3 \mathrm{~cm}$ of the major vessel was the predominant site of calcification in all cases, and there were no cases of calcification or peripheral vessels without proximal involvement. Pathological examination revealed a similar distribution of atheromatous lesions.

\section{Aortic valve calcification}

By using the air-filled shadow of the ascending aorta as a guide the aortic valve was easily identified in the radiographs. In 16 cases calcification was detected in the aortic valve mainly at the junction of the cusps and valve ring. In only one had there been clinical evidence of aortic valve disease. In all but one of the 16 cases aortic valve calcification was associated with coronary artery calcification (93\%). There is, therefore, a strong relation between aortic valve sclerosis and coronary calcification (Fig. 3a).

\section{Mitral valve calcification}

Five cases showed calcification in the mitral valve cusps or annulus. There was no correlation with coronary calcification. Care was required to differentiate mitral annulus calcification from artery calcification in the radiographs (Fig. 3a and 4).

\section{Correlation with pathological findings}

Each heart was carefully examined microscopically and areas of apparent abnormality in the coronary arteries were removed for section. Areas suggested as abnormal on the radiographs were also removed.

The blocks of excised tissue were embedded in gelatin which were then frozen and sections cut on a freezing stage microtome at $5-7 \mu$ and floated on glass slides. Individual sections were stained to demonstrate fat, muscle cells, fibrin, mucopolysaccharide, elastic tissue, and iron. A detailed study of the disease process could thus be made (McCarthy, 1973).

For the purpose of histological correlation the cases could be divided into three groups according to the radiographic findings.

A) No radiographic abnormalities (I4 cases) All cases showed some evidence of histological abnormality even in the paediatric age group. The initial lesion of atheroma consisted of disruption of the internal elastic lamina with proliferation of muscle cells into the intima. Stains for lipid and cholesterol showed no evidence of these substances in this type of lesion. 


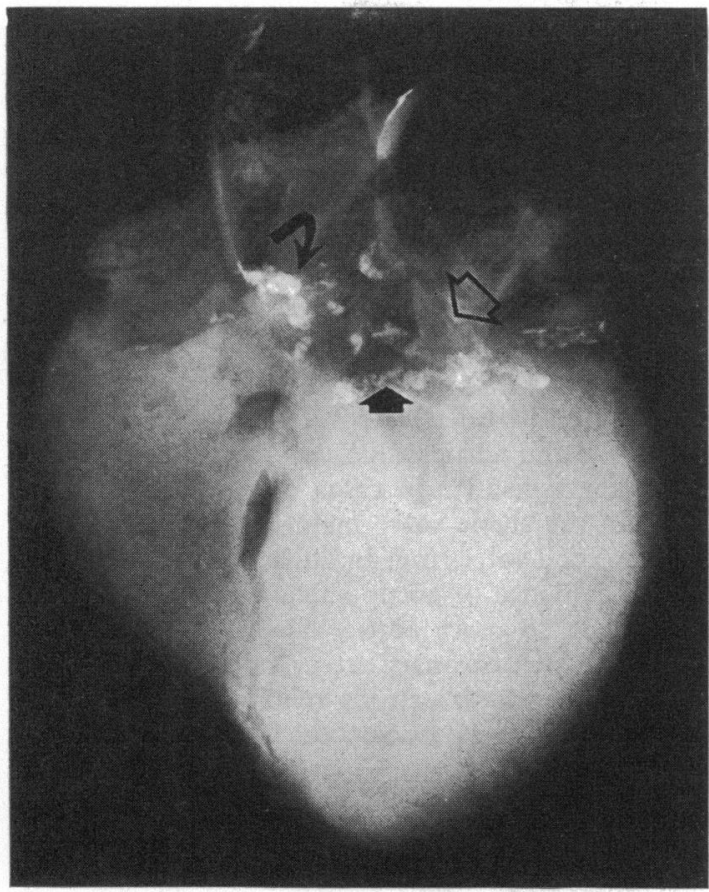

(a)

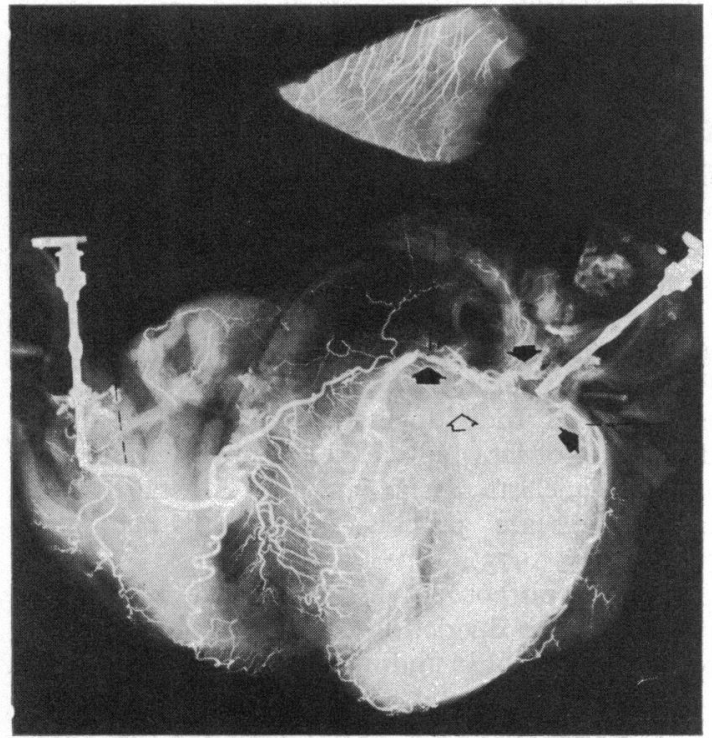

(b)

F I G. 3 a) Aortic valve calcification (curved arrow), mitral valve calcification (closed arrow), and coronary artery calcification (open arrow). b) Mitral valve calcification again seen (open arrow). Tight stenosis in anterior descending and circumflex arteries at sites of calcification.
B) Radiographic lumenal irregularity without calcification (10 cases) Similar but more pronounced changes were found. There was considerable fragmentation of the internal elastic lamina with pronounced proliferation of fibroblasts and muscle cells within a thickened intima. Localized area of such fibromuscular plaques produced narrowing of the vessel lumen, which correlated well with the arteriographic appearances. Collagen and lipid could be detected in the intima at these sites but there was no evidence of cholesterol.

C) Calcification of vessels of varying severity (4I cases) All cases showed some degree of lumenal irregularity, varying from minimal vessel narrowing to complete occlusion. Calcification was confirmed microscopically in all cases where it was seen radiologically.

Two types of calcification were found: I) Small deposits in fibromuscular plaques close to the internal elastic lamina and without associated necrosis. These were always found in such atheromatous areas and were associated with minimal narrowing radiographically.

2) Large deposits $(>0.5 \mathrm{~cm})$ associated with frank necrosis within the intima. In these lesions the internal elastic lamina had largely disappeared and the media degenerated. The necrotic area contained considerable quantities of lipid and cholesterol. These lesions were associated with more pronounced vessel narrowing $(>70 \%)$, and rupture of the necrotic material into the vessel lumen could be identified microscopically in some cases.

The relative importance of degeneration of fibromuscular plaques and inundation of lipid and cholesterol from the blood stream was not determined by this study.

Pronounced calcification was associated in all cases with severe atheromatous disease in the involved vessel. Thus, all cases with calcification extending for over $\mathrm{I} \mathrm{cm}$ of a vessel or with block calcification of several vessels demonstrated angiographic evidence of significant vessel stenosis or frank occlusion (Fig. 4a and b).

In 19 hearts vessel occlusion was demonstrated angiographically and confirmed by dissection and microscopy. In 16 of these calcification was present in the vessel wall at the site of occlusion (Fig. 2a, Fig. 3a and b, Fig. 5a and $b$ and Fig. 6). The three cases without associated calcification were of a comparatively younger age (44,54, and 58 years), and pathological examination revealed recent acute coronary thrombosis. 


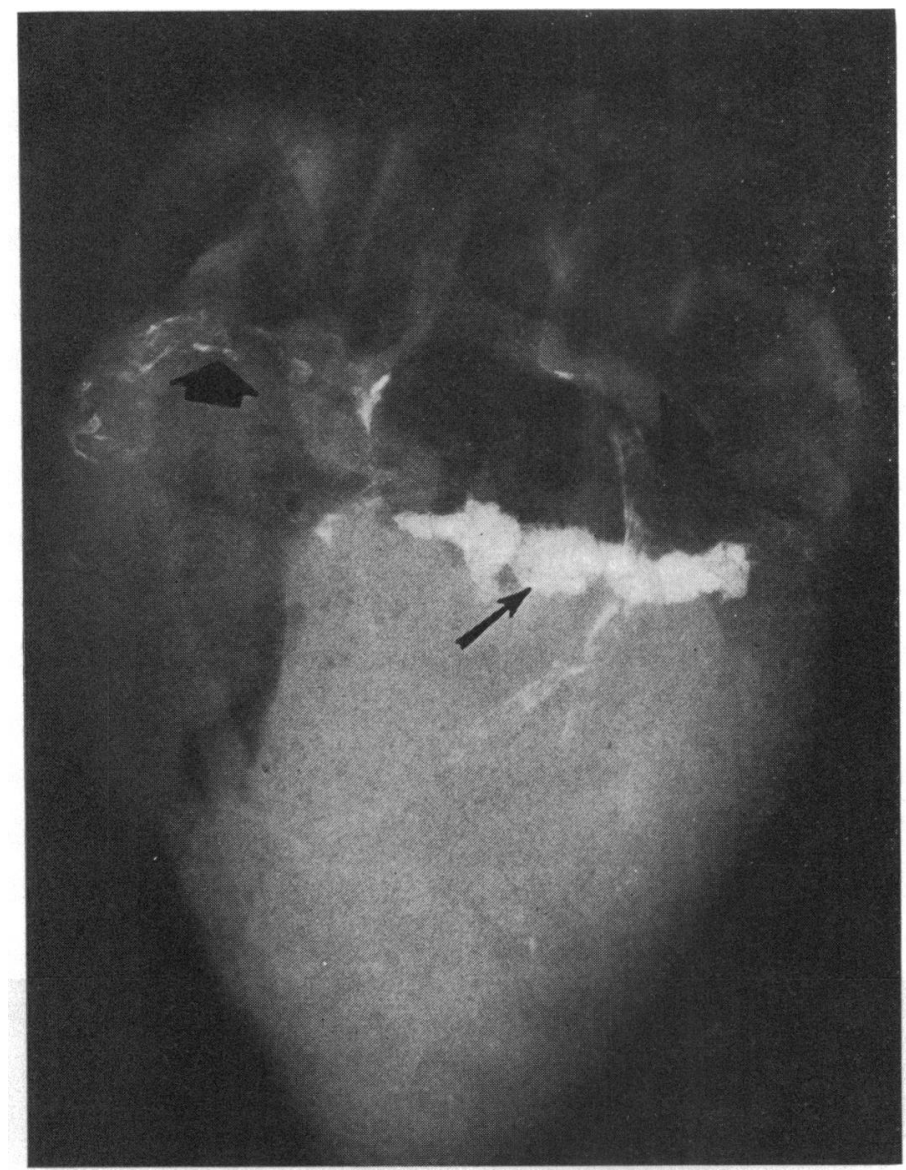

FI G. 4 Gross calcification in annulus of mitral valve (thin arrow). Double calcification in right coronary artery (wide arrows).

This study demonstrates a clear relation between calcification and arterial atheroma. Calcified lesions of the order visible by fluoroscopy, i.e. $0.5 \mathrm{~cm}$ (Blankenhorn and Stern, 1959) are associated with necrotic lesions of the intima and vessel narrowing. The degree of vessel stenosis and the incidence of complete vessel occlusion correlates well with the extent of radiologically visible calcification.

\section{Discussion}

Calcification in the wall of an involved vessel is commonly demonstrated in atheromatous lesions throughout the arterial system. Coronary artery atheroma similarly undergoes calcification but the significance of this is disputed. It has been suggested that calcification is a measure of the age of the atheromatous process and that it often occurs in elderly arteries without significant lumenal obstruc- tion (Vlodaver et al., 1972). Other authorities have ventured similar opinions (Adams et al., 1972).

Certainly the correlation with clinical cardiac ischaemia is variable, dependent as it is on such factors as the degree of vessel stenosis, distribution of lesions, arterial anatomical variations, and the development of collateral circulation. However, this study has demonstrated that, even in the elderly, pronounced coronary artery calcification is associated with severe atheroma and vessel stenosis above or approaching the critical level of 50 to 75 per cent reduction of vessel calibre or frank vessel occlusion. There have been several studies made using methods similar to the present investigation whose authors have also concluded that there is a strong correlation (Beadenkopf, Daoud, and Love, 1964; Blankenhorn and Stern, 1959; Eggen, Strong, and McGill, 1965; Jorgens et al., I965; 


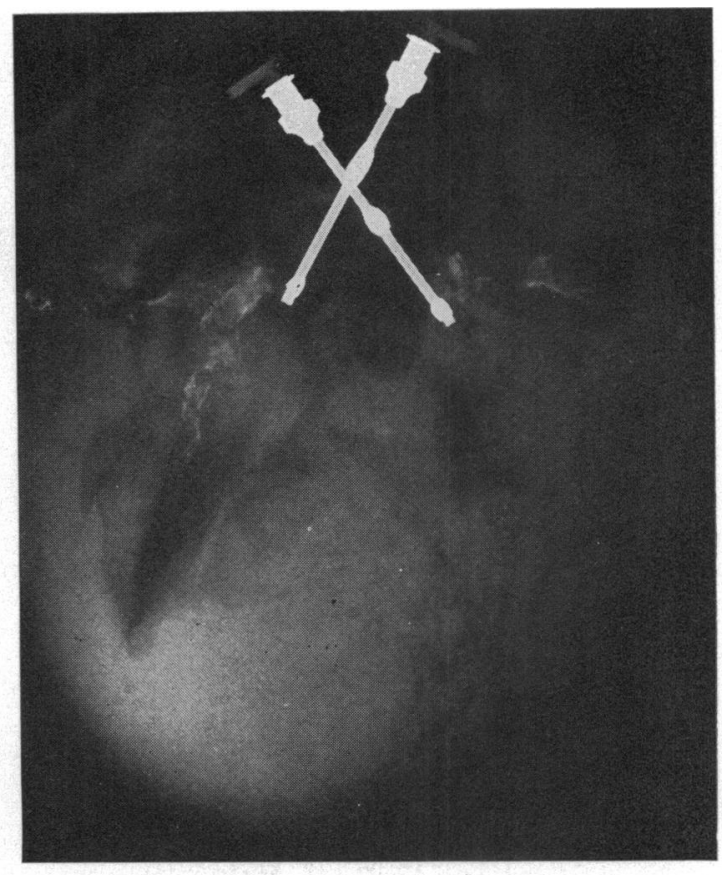

(a)

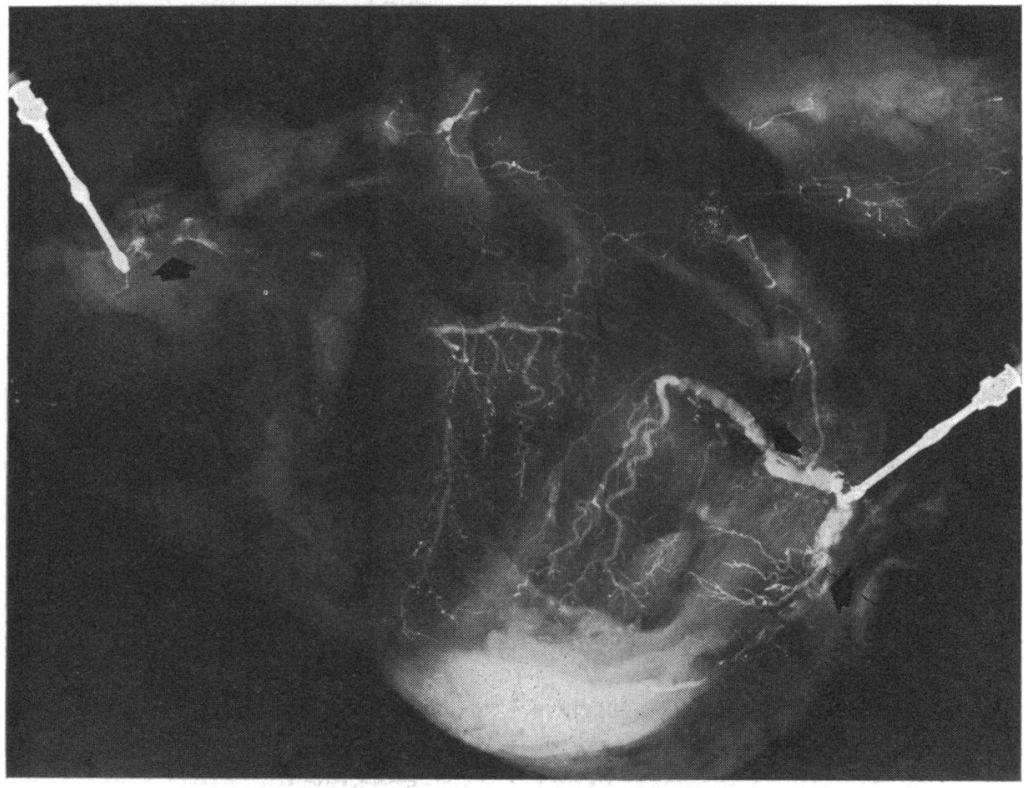

(b)

FIG. 5 a) Calcification in right coronary, anterior descending, and circumflex vessels. b) Complete occlusions in corresponding vessels demonstrated by contrast injection (wide arrows). Note the collateral vessel supplying the distal circumflex artery (thin arrow). 


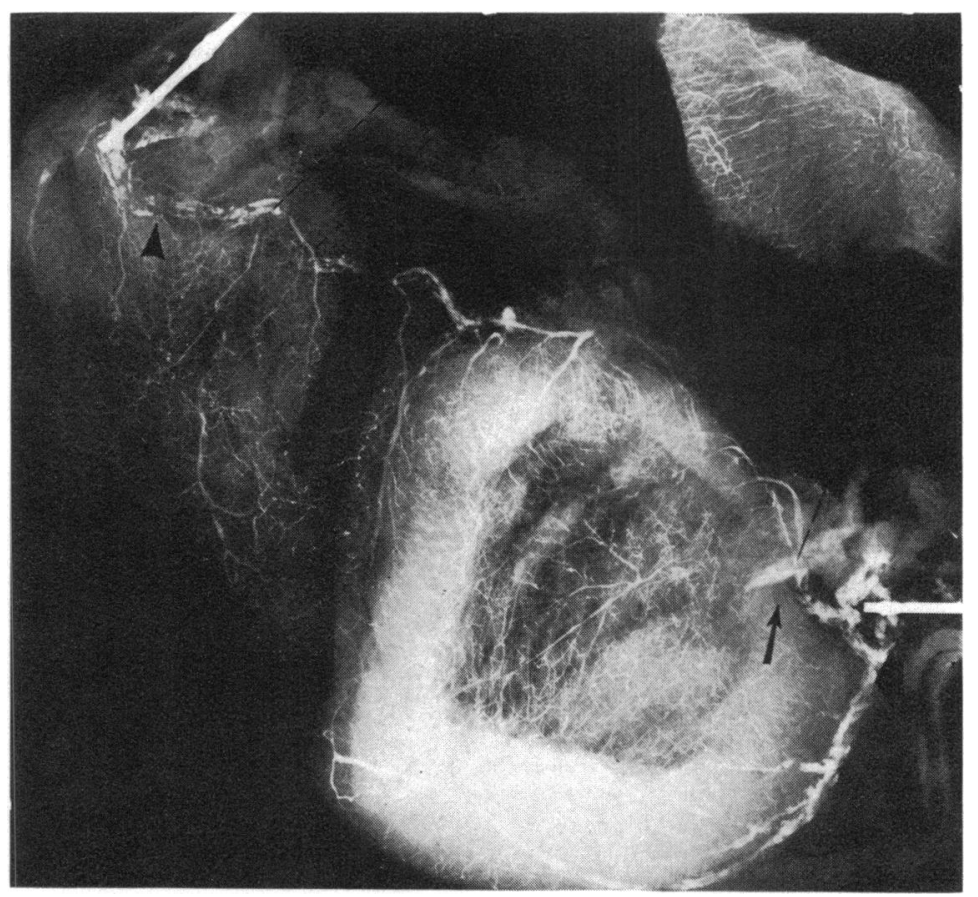

FIG. 6 Complete occlusions in right artery and circumflex arteries (arrows). Stenosis also seen in anterior descending artery. The vessels were heavily calcified on the plain film.

Tampas and Soule, 1966; Oliver et al., 1964). It is therefore our contention that patients having pronounced coronary calcification are at risk from coronary occlusion, including those without clinical evidence of ischaemic heart disease. Coronary calcification is therefore of important prognostic significance.

Osborn (1963) states, 'we have no means of saying whether the coronary arteries of the man in the street are good, bad or indifferent'. Screening of the heart for coronary calcification at routine fluoroscopy is a simple procedure that gives some measure of the subclinical coronary atheroma in the general population. This finding in a comparatively young patient should lead to further investigation.

Calcified lesions of the order of $0.3 \mathrm{~cm}$ diameter are theoretically visible fluoroscopically (Blankenhorn and Stern, 1959). Random measurements of lesions in our material show that the majority of calcifications associated with stenosis obey these criteria.

Many patients now attending hospitals are presently examined by image intensified fluoroscopy during investigation of respiratory, cardiac, gastrointestinal, urological, or vascular problems. Examination of the cardiac silhouette for coronary calcification can be incorporated in these examinations without significantly increasing the duration of the procedure or the amount of radiation. We support the plea (Tampas and Soule, 1966; Warburton et al., 1968; Oliver, 1970) that a search for coronary calcification should be included in all such fluoroscopic procedures.

\section{References}

Adams, D. F., Abrams, H. L., and Ruttley, M. (1972). The roentgen pathology of coronary artery disease. Seminars in Roentgenology, 7, 319.

Beadenkopf, W. G., Daoud, A. S., and Love, B. M. (1964). Calcification in the coronary arteries and its relationship to arteriosclerosis and myocardial infarction. American fournal of Roentenology, 92, 865.

Blankenhorn, D. H., and Stern, D. (1959). Calcification of the coronary arteries. American fournal of Roentgenology, 81, 772.

Eggen, D. A., Strong, J. P., and McGill, H. C. (1965). Coronary calcification: relationship to clinically significant coronary lesions and race, sex, and topographic distribution. Circulation, 32, 948.

Jorgens, J., Boardman, W. J., Damberg, S. W., Kinney, W. N., and Kundel, R. R. (1965). The significance of coronary calcification. American fournal of Roentgenology, 95, 667.

Laurie, W., and Woods, J. D. (1958). Anastomosis in the coronary circulation. Lancet, 2, 812.

McCarthy, J. H. (1973). The nature of the occlusion in coronary artery disease. Thesis for B.Sc., University of New South Wales.

Oliver, M. F. (1970). The diagnostic value of detecting coronary calcification. Circulation, 4I, 981 . 
Oliver, M. F., Samuel, E., Morley, P., Young, G. B., and Kapur, P. L. (1964). Detection of coronary-artery calcification during life. Lancet, $1,891$.

Osborn, G. R. (1963). The Incubation Period of Coronary Thrombosis. Butterworths, London.

Schlesinger, M. J. (1938). An injection plus dissection study of coronary artery occlusions and anastomoses. American Heart fournal, 15, 528.

Sutton, D., and Grainger, R. G. eds. (1969). A Textbook of Radiology, E. S. Livingstone, Edinburgh.

Tampas, J. P., and Soule, A. B. (1966). Coronary artery calcification. American fournal of Roentgenology, 97, 369.
Warburton, R. K., Tampas, J. P., Soule, A. B., and Taylor H. C. (1968). Coronary artery calcification: its relationship to coronary artery stenosis and myocardial infarction. Radiology, 91, 109.

Vlodaver, Z., Neufeld, H. N., and Edwards, J. E. (1972). Pathology of coronary disease. Seminars in Roentgenology, 7, 376.

Requests for reprints to Dr. F. J. Palmer, Department of Radiology, Prince Henry Hospital, Little Bay, N.S.W. 2036, Australia. 